${ }^{1}$ Head of the Department of Surgical Dentistry, Maxillofacial Surgery and Oncostomatology, Uzhhorod National University, Ukraine

${ }^{2}$ Head of Scientific and Research Centre of Forensic Odontology, Department of Prosthetic Dentistry, Uzhhorod National University, Ukraine.
Corresponding author: Myroslav Goncharuk-Khomyn Uzhhorod National University Universitetska 16/a st., Uzhhorod Ukraine

Area code: 88000

Phone number: 0991212813

E-mail address: myroslav. goncharuk-khomyn@uzhnu.edu.ua

Received: June 04, 2020

Accepted: November 23, 2020

\section{Economical treatment- related burden assessment of maxillofacial trauma among Ukrainians patients}

\author{
Pavlo Brekhlichuk ${ }^{1}$ iD, Myroslav Goncharuk-Khomynn ${ }^{2, *}$ iD
}

Aim: Quantitative evaluation of prognostic correspondence between initial maxillofacial traumatic injury assessed by facial injury severity score and maxillofacial injury severity score, treatment cost and duration of hospitalization among Ukrainian patients. Methods: Design of present study was retrospective and based on the medical data of patients hospitalized with signs of maxillofacial trauma. Quantitative assessment of maxillofacial trauma was held with the use of facial injury severity score (FISS) and maxillofacial injury severity score (MFISS). Average treatment cost and hospitalization duration were used as coordinative criteria for economical treatmentrelated burden verification. Results: Levels of correlation between FISS, treatment charges and hospitalization duration were $r=0.69(p<0.05)$ and $r=0.67 \quad(p<0.05)$ respectively, while analogical correlations for MFISS were $0.74(p<0.05)$ and 0.69 respectively $(p<0.05)$. Statistical correspondence between FISS and MFISS scores among study sample reached $r=0.71$ $(p<0.05)$. Cases with milder maxillofacial trauma types, characterized with initial lower levels of FISS and MFISS scores, demonstrated greater degree of FISS-to-MFISS inter-relation compare to cases with severe maxillofacial trauma. Conclusion: Even though FISS and MFISS scores both demonstrated reliable levels of correlation with hospitalization duration and cost of dental rehabilitation after maxillofacial trauma injury, but MFISS approach characterized by prognostically greater level of statistical relationship with economically related treatment derivates. Moreover, differentiation capabilities of MFISS is relative greater than FISS, since independent grading of separate functional disabilities becomes possible.

Keywords: Maxillofacial injuries. Treatment outcome. Cost of illness. Hospitalization. 


\section{Introduction}

Relevant predictive models of dental care supply, especially under the conditions of different insurance policies, should be based on the valid quantitative approaches aimed at primary differentiation and categorization of maxillofacial injuries in terms of needed dental interventions, their cost and treatment efficiency ${ }^{1-3}$. Nevertheless, most of the used insurance calculation protocols considering the fact of maxillofacial trauma and it's situational parameters as main criteria for future prognosis regarding payments of claims ${ }^{2-5}$. Argumentative choice of economically-available and predictively-effective treatment modality could help to optimize overall rehabilitation process among maxillofacial trauma patients considering initial injuries of anatomical structures and associated functional alterations ${ }^{2,6,7}$. Rapid scoring protocol of maxillofacial trauma also could provide further perspectives for primary patients categorization within trauma center conditions ${ }^{1,7,8}$.

Due to the number of previously published studies it is argumentative to resume that maxillofacial injury severity scoring system (MFISS) and facial injury severity scoring system (FISS) are considered as ones of the most prevalent among researchers' use $^{1,6,7,9-12}$, while in one of the studies such conclusion even was solidly clarified ${ }^{2}$. Taking into account original scoring methodologies of MFISS and FISS scores and available literature data on their use with research objective, the first one is considered to be more functionally-oriented, while second - anatomically-based $d^{1,2,6,7,9-11}$. On the other hand number of publications revealed that both of these scores demonstrated comparatively analogical statistical associations with treatment duration, rehabilitation charges, injury severity, complication rates and some other parameters 1,2,7,10-12.

In multicentered study of European Maxillofacial Trauma it was noted that even though FISS scores were relatively analogical by the mean values among different centers, the longest hospitalization durations were noted in Kiev (Ukraine) ${ }^{13}$. Since treatment methods and primary post-traumatic care differ among different countries, even though such are following the same biological and medical principles, it is important to find out how the FISS and MFISS scores predictively relate with the regionally-specific economic burden parameters of hospitalization and rehabilitation, thus widening the perspective of their use with an aim of dental care and insurance support optimization.

Considering all above-mentioned facts, our research was aimed at the quantitative evaluation of prognostic correspondence between initial maxillofacial traumatic injury assessed by FISS and MFISS scores, treatment cost and duration of hospitalization among Ukrainian patients.

\section{Materials and methods}

Design of present study was retrospective and based on the medical data of patients hospitalized to the Uzhhorod City Clinical Hospital (Uzhhorod, Ukraine) with signs of maxillofacial trauma during 2015-2019. Study sample among all received patients' data sets was formed due to the next inclusion criteria: 1) preliminary diagnosis of maxillofacial trauma provided at the time of hospitalization; 2) accessibility of full trauma characteristics description in provided medical documentation; 3) presence of supplemental $\mathrm{X}$-ray diagnostics results or their full interpretations inside medical 
documentation; 4) availability of full description regarding provided treatment with exact postscript of hospitalization duration and cost of treatment. Exclusion criteria presented by the next parameters: 1) absence of necessary information related to the diagnostic process, anamnesis, provided treatment, cost or hospitalization duration; 2) concomitant traumatic injury; 3) combined trauma with related head and neck, eye-ball injury or neurotrauma; 4) compromised anamnesis with allied somathopathologies that potentially could alter rehabilitation process ${ }^{14}$. Due to the inclusion and exclusion criteria group of 65 patients with maxillofacial trauma was formed.

All patients were treated at the same accident and emergency department with further admission to the in-patient facility. Data extraction was provided considering anonymization and ethical principles with further analysis of only next parameters: age, gender, characteristics of trauma, duration of hospitalization, average treatment cost ${ }^{15}$. Average treatment cost was accounted by provisional monetary units due to the provided diagnostics complex, dental surgical rehabilitation, hospitalization and in-patient care without considering cost of pharmacological support. Hospitalization duration was defined by the period between primary patient's admission to the hospital till the official discharge ${ }^{2}$. Such criteria as average treatment cost and hospitalization duration were used as coordinative for economical treatment-related strain verification for each patient from study group².

Quantitative assessment of maxillofacial trauma was held with the use of facial injury severity score (FISS) and maxillofacial injury severity score (MFISS). Evaluation was provided due to the original protocols of such scoring systems proposed by Bagheri et al. and Zhang et al. respectively, ${ }^{6,9}$. Use of MFISS and FISS scores as comparable and referent for analytical prognosis considering average treatment cost and duration of hospitalization, was argumented by high level of such criteria correlation with expert maxillofacial injury evaluation, described in previous studies ${ }^{1,2,12}$. Assessment of FISS and MFISS scores was provided by two independent investigators (members of Scientific and Research Center of Forensic Odontology, Uzhhorod National University), who were previously calibrated with the use of reference data and characterized with inter-observer agreement of $k=0.81$.

Design of provided study was previously approved by ethical committee of Faculty of Dentistry (Uzhhorod National University) as a part of the complex research related to the clinical and laboratorial assessment of advanced dental technologies and expert evaluation of treatment methods (Ethical Approval № 25072017-13).

Exploratory data analysis principles were used for study sample characterization with the evaluation of above-mentioned criteria (age, gender, characteristics of trauma, duration of hospitalization, average treatment cost). Univariative statistical analysis with estimation of mean, maximum and minimum was provided considering criteria of FISS and MFISS scores independently among study group patients. Pearson's r was used for the estimation of correlation between FISS and MFISS scores and such parameters as average cost of treatment and hospitalization duration, while probability value ( $p$-value) lower than 0.05 was considered as statistically reliable. Student's t-test was used for assessment of statistical difference between MFISS and FISS criteria patterns among study group patients, and distinction trends of their relation to the average treatment cost and hospitalization duration ${ }^{16,17}$. Bland and Altman analysis was provided with XLSTAT 2020 software tool (xlstat.com) ${ }^{18,19}$. Stratification of data and its' further graphical representation were provided via Microsoft Excel software (Microsoft Office, 2019). 


\section{Results}

Distribution of maxillofacial trauma patients sample by the age and gender criterions was presented as following: out of 65 subjects 53 (81.54\%) were males and 12 (18.46\%) were females with ratio of $4.41: 1$; 18 persons (27.69\%) were within age group of 20-30 years (mean age -26.72 years), 37 (56.92\%) - within age group of 30-40 years (mean age - 34.54 years), 10 persons (15.38\%) - within age group of 40-50 years (mean age 46.31 years). Obtained distribution tendencies partially promoted by used specific inclusion criteria.

Main causes of maxillofacial trauma among study sample were presented by interpersonal violence (assaults) - 29 patients (44.62\%), road-traffic accidents - 22 patients (33.85\%), falls -9 patients (13.85\%), work-associated and sport-related -5 patients (7.69\%). Most cases of interpersonal violence, road-traffic accidents and work/ sport-related traumas as causes of maxillofacial trauma were registered among male patients $(65.52 \%, 63.64 \%$ and $60.0 \%$ respectively), while most cases of falls $(55.56 \%)$ were noted among females. Among all 65 analyzed cases $47(72.30 \%)$ of them were presented with bone fractures (mandible fractures - 14 cases $(21.54 \%)$, Le Fort I fractures -5 cases (7.69\%), Le Fort II fractures - 4 cases (6.15\%), Le Fort III - 4 cases (6.15\%), zygoma complex fractures -6 cases (9.23\%), nasal fractures - 10 cases (15.39\%), orbital fractures -4 cases $(6.15 \%)$ ), while in 18 cases $(27.69 \%)$ such were also associated with pronounced adjacent soft tissue injuries (projected mostly at the lower one third of the face in 7 cases (10.77\%), at the mid-face area - in 6 cases (9.23\%), and at the upper one third - in 5 cases (7.69\%), while in most cases lacerations projected beyond restricted area of some one third part of the face).

Numerical results received during FISS and MFISS scoring were characterized with normal distribution pattern, which also was described in previous study ${ }^{1}$. Mean FISS score for study

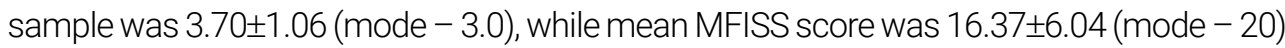
(Table 1). Average period of hospitalization duration was equal to $8.9 \pm 2.4$ days.

Table 1. FISS and MFISS scores statistical characteristic registered among study sample

\begin{tabular}{lccccccc}
\hline Variable & Observations & $\begin{array}{c}\text { Obs. with } \\
\text { missing data }\end{array}$ & $\begin{array}{c}\text { Obs. without } \\
\text { missing data }\end{array}$ & Minimum & Maximum & Mean & $\begin{array}{c}\text { Std. } \\
\text { deviation }\end{array}$ \\
\hline FISS & 65 & 0 & 65 & 1.000 & 6.000 & 3.708 & 1.057 \\
\hline MFISS & 65 & 0 & 65 & 5.000 & 30.000 & 16.369 & 6.035 \\
\hline
\end{tabular}

Levels of correlation between FISS, average treatment charges and hospitalization duration were $r=0.69(p<0.05)$ and $r=0.67(p<0.05)$ respectively, while analogical correlations for MFISS were $0.74(p<0.05)$ and 0.69 respectively $(p<0.05)$ (Table 2$)$.

Table 2. Correlation level between FISS, MFISS, hospitalization duration and cost of treatment

\begin{tabular}{lcccccc}
\hline Criteria & $\begin{array}{c}\text { Cost of } \\
\text { treatment }\end{array}$ & p-value & $\begin{array}{c}\text { Hospitalization } \\
\text { Duration }\end{array}$ & p-value & FISS & p-value \\
\hline FISS & 0.69 & $\mathrm{p}<0.05$ & 0.67 & $\mathrm{p}<0.05$ & 1.0 & $\mathrm{p}<0.05$ \\
\hline MFISS & 0.74 & $\mathrm{p}<0.05$ & 0.69 & $\mathrm{p}<0.05$ & 0.71 & $\mathrm{p}<0.05$ \\
\hline
\end{tabular}

Statistical correspondence between FISS and MFISS scores among study sample reached $r=0.71(p<0.05)$ (Fig. 1). 


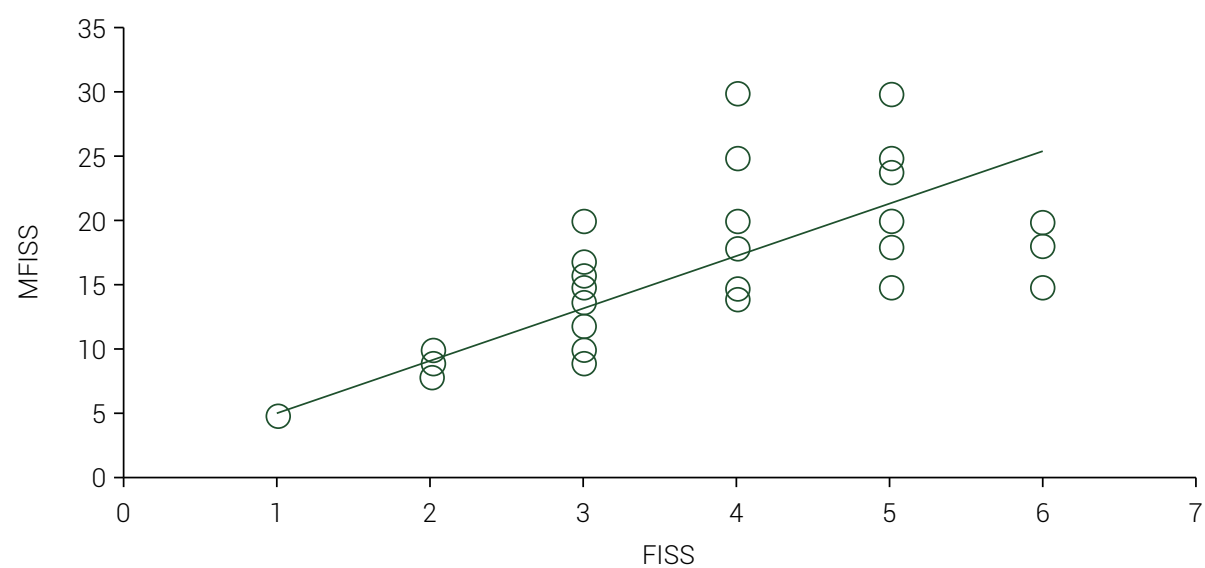

Figure 1. Correlation between FISS and MFISS scores registered among study sample.

Without preliminary standardization of obtained data statistical difference was noted during pairwise comparison of FISS and MFISS results during analysis of 57 (87.69\%) individual cases. Patients with FISS scores greater than 3 and MFISS scores greater than 10 were characterized with statistically longer period of hospitalization compare to study subjects with lower obtained scores levels $(p<0.05)$.

Results of Bland-Altman analysis considering relationship between FISS and MFISS scores represented on the Figures 2-4, with primary received data presented in Table 3.

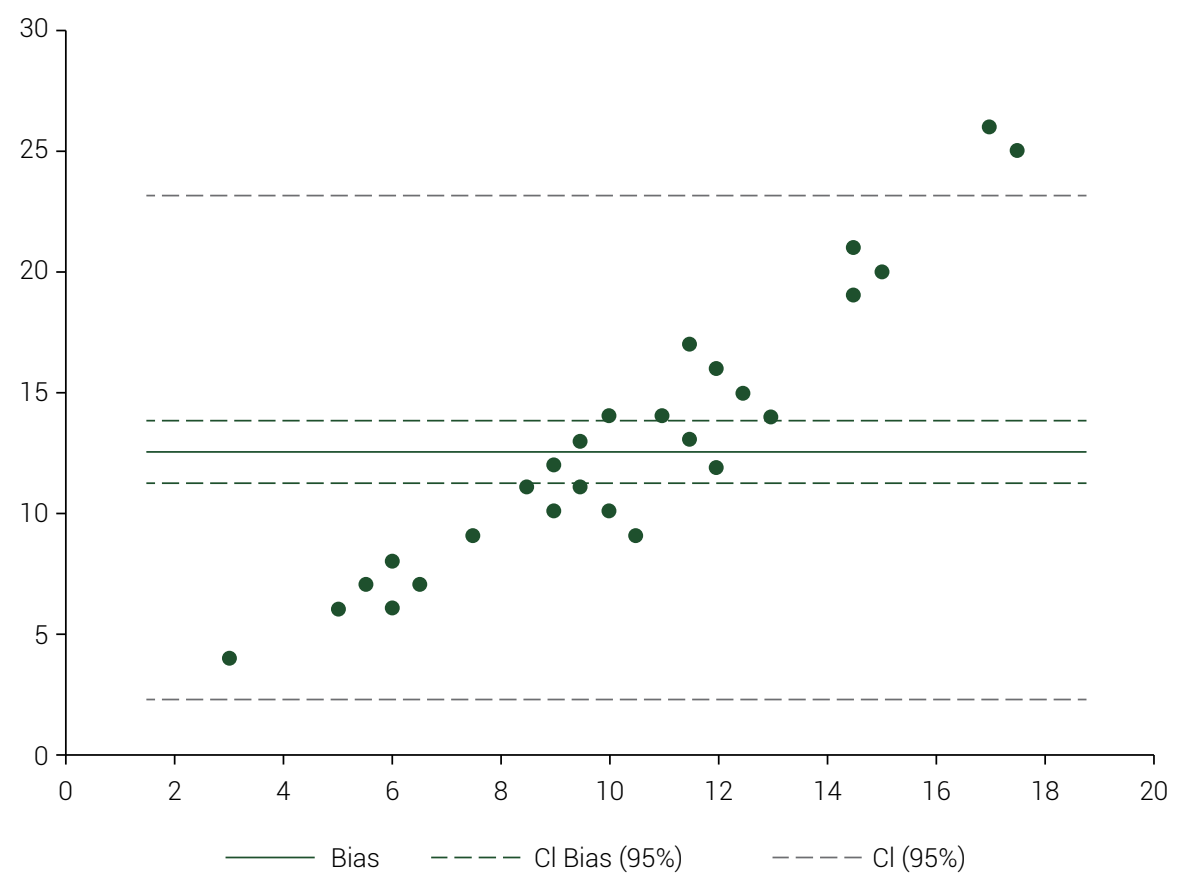

Figure 2. Bland-Altman plot for FISS and MFISS scoring results 


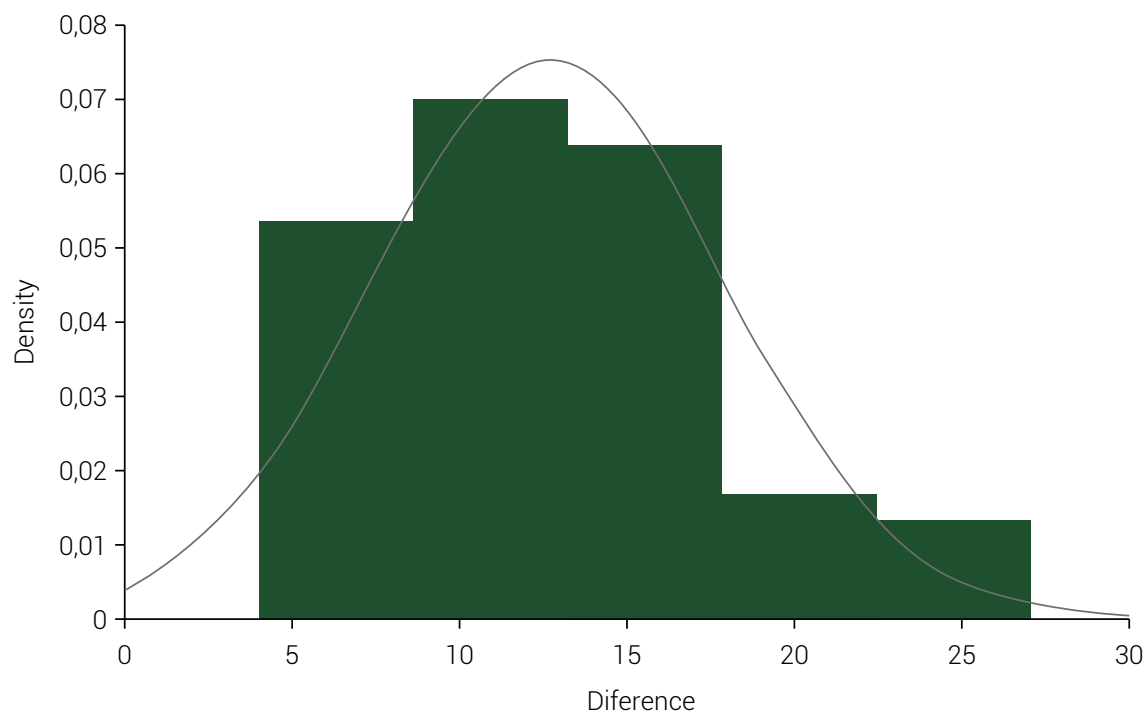

_ Diference _ Normal

Figure 3. Distribution of differences in FISS/MFISS scores due to the normality assumption

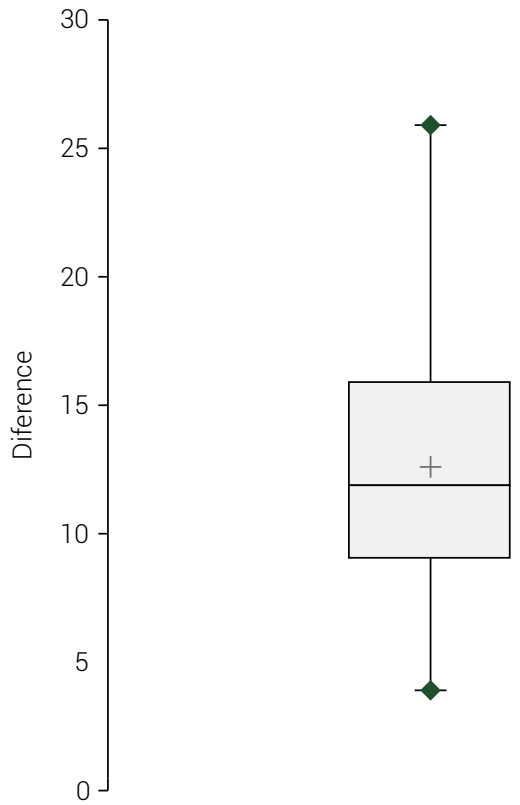

+ Mean

Minimum/Maximum

Figure 4. Correspondence between mean and median of FISS/MFISS due to the Bland-Altman analysis 
Table 3. Data received during Bland-Altman analysis of MFISS and FISS score

\begin{tabular}{lccccc}
\hline \multirow{2}{*}{ Bias } & \multirow{2}{*}{ Standard error } & \multicolumn{2}{c}{ Cl Bias (95\%) } & \multicolumn{2}{c}{ Confidence interval (Differences): } \\
\cline { 2 - 5 } & & Lower Limit & Upper Limit & Lower Limit & Upper Limit \\
\hline 12.66 & 5.33 & 11.33 & 13.98 & 2.19 & 23.12 \\
\hline
\end{tabular}

Obtained results shown that both scores are analogically effective from the evaluation point of view and could be used for maxillofacial trauma assessment considering treatment expenses and hospitalization duration as targeted research parameters. But specific pattern of FISS/MFISS relationship was noted, due to which cases with milder maxillofacial trauma types, characterized with initial lower levels of FISS and MFISS scores, demonstrated greater degree of FISS-to-MFISS statistical inter-relation compare to cases with severe maxillofacial trauma.

\section{Discussion}

The variability of the maxillofacial traumatic lesions cases and the prevalence of such among able-bodied persons justify the need for investigation, development and improvement of expert evaluation approaches considering dental changes of primary traumatic and secondary iatrogenic origin ${ }^{13,20-22}$. In present research we have argument the correlational levels between obtained FISS/MFISS scores, which indirectly related to the anatomical and functional severity of maxillofacial trauma, and economical parameters of provided dental care in means of average treatment cost and hospitalization duration. The results of previous analytical studies indicated the presence of relationship between the necessary amount of dental rehabilitation interventions and initial characteristics (location, severity, spread) of obtained dental injuries 1,2,13,20. $^{2}$.

Due to the EURMAT project data the most prevalent causes of maxillofacial trauma were assault and falls ${ }^{13}$, while in our study most of trauma injuries were caused by interpersonal violence and road-traffic accidents. Falls and work-associated/sport-related injuries were third and fourth the most prevalent causes of patients' hospitalization with maxillofacial trauma. Analogically to EURMAT project data related to Ukraine, male/female ratio in our study also was characterized with predominant number of male patients compare to female (4.41:1), while overall European ratio was at level of 3.6 to $1^{13}$. Similarly, to the findings noted by Siber et al. $(2015)^{20}$, we have also registered the bone injuries as the most prevalent among study sample of patients with maxillofacial trauma.

Providing retrospective study Bocchialini and Castellani (2019) had found that increase of FISS score parameter by 1 point associated with the increase in hospitalization duration on $12 \%$ ( 1.44 days $)^{23}$. More pronounced relationship between FISS score and length of hospital stay was described in Siregar et al. study (2019) ${ }^{24}$. Authors mentioned that increase of FISS at the level of more than 3 characterized with 14 times more chances of longer hospitalization ${ }^{24}$. Under the conditions of retrospective study, it was found the FISS scores greater than 5 causing 18 times more chances to be hospitalized compare to the situations when FISS score was lower than 6, while FISS scores greater than 5 were also statistically associated with need of minimum 3 days hospitalization $(P<0.01)^{25}$. Other researchers highlighted that level of FISS $\leq 3$ was 
relevant for cases of maxillofacial trauma hospitalization up to 6 days, while FISS level of greater than 12 in most cases caused hospitalization for more than 10 days ${ }^{23}$. Bagheri himself as an author of FISS scoring system mentioned that even though such demonstrated statistical association with the hospitalization duration, but it could not be categorized as fully reliable predictor ${ }^{9}$. In his research 3 cases of death were highlighted, while non-survivors' FISS scores were not statistically different from those registered among survivors ${ }^{9}$. Nevertheless, in all above-mentioned lethal cases victims demonstrated FISS scores greater compare to average ones noted during analysis ${ }^{9}$. Considering variability of FISS/MFISS scores and hospitalization duration, we could not register some specific pattern of correspondence between 1 FISS/ MFISS point increase and additional number of days with needed in-patient care, but it was found that FISS scores greater than 3 and MFISS scores greater than 10 associated with more prolonged period of hospitalization compare to situations with lower obtained scores levels, difference between which was statistically approved $(p<0.05)$.

Analogical to our, study was provided also by Ramalingam S. (2015), who have found out that both MFISS and FISS scores were characterized with relatively equal correlation due to the cost ( $r=0.862$ and $r=0.845$ respectively) and duration of hospitalization $(r=0.828$ and $r=0.819)$ among Indian population ${ }^{2}$. Considering such results author highlighted the role of MFISS and FISS as "economic burden" indices, while in our study FISS demonstrated lower correlation levels with duration of hospitalization and the cost of treatment ${ }^{2}$. The presence of analogical study gives us a unique possibility to analyze potential causes of obtained results dissimilarities. Such could be provoked by the influence on next factors: different approaches of patients stratification in India and in Ukraine; different calibration levels of dental experts, who provided the evaluation of patients regarding MFISS and FISS criteria; different distribution of costs for specific dental trauma treatment algorithm in India and Ukraine; variances of national currency due to the standardized cost of dental treatment calculated by insurance companies in means of provisional monetary units; differences of samples sizes.

Analogical to our findings, such also were described in Giriyan et al. (2019) study, in which authors had registered Spearmen's correlation levels of $r=0.398$ and $r=0.429$ between MFISS/FISS values and treatment cost respectively, and correlation levels of $r=0.477$ and $r=0.433$ between MFISS/FISS values and hospitalization time respectively ${ }^{12}$. While estimated levels of correlation were lower compare to those in Ramalingam's study², they were statistically approved. Similarly to previous study, it was found that in cases of mid-face fracture among Chinese population MFISS and FISS scores represent statistically analogical interrelations with hospitalization duration $-r=0.415$ and $r=0.464$ respectively ${ }^{26}$. Moreover, authors found out statistically reliable dependencies between FISS scores and gender, age, etiology and fracture type parameter, while MFISS scores demonstrated connection with gender at the $p=0.201$ and with age at $p=0.052$. Nevertheless, MFISS and FISS scores correlated between themselves at $r=0.0592(p=0.01)^{26}$. In our study relationship between above mentioned parameters (gender, age, etiology and fracture type) were out of primary formulated objective, while they will be considered as a perspective for future research

It is interesting to note that in the comparative study of different maxillofacial trauma grading approaches, FISS scores demonstrated the lowest level of correlation with 
expert evaluation results ( $r=0.699)$, while MFISS demonstrated the greatest $(r=0.801)$ among all studied scoring systems ${ }^{1}$. Despite that FISS scores were characterized by statistically the highest interrelation pattern with the cost of operation $(r=0.742)$, while correlation levels of FISS $(r=0.620)$ and MFISS $(r=0.636)$ scores were comparable due to the operation time parameter ${ }^{1}$. Our results are partially consistent with those described by Chen et al. (2014) ${ }^{1}$ in terms, that in our study MFISS scores also have shown the highest level of correlation not only with duration of hospitalization, but also with the average cost of treatment.

Originally FISS was described as anatomically-based by the methodology of calculation in many of previously published studies ${ }^{1,2,7,10-12}$, but considering specific categorization of such grading trauma system, we could resume that this criteria is also partially functionally-oriented, even if such traumatic functional association is not so directly represented, as in MFISS methodology. For example, Le Fort III fracture gains greater score than Le Fort I or Le Fort II, which is logical, because such fracture is causing greater anatomical disruption, but it should be noted that Le Fort III fracture is also associated with more pronounced functional alterations. So, we would propose to classify FISS scoring system as "predominantly anatomically-oriented", rather than just "anatomically-based".

Considering today's progress in maxillofacial surgery and forensic dentistry new scoring systems for maxillofacial trauma evaluation still developing. Canzi and colleagues described comprehensive facial injury (CFI) score, which differs by high descriptive capacity and with this characteristic supports patient differentiations in trauma centers $^{27,28}$. Potentially $\mathrm{CFI}$ could be used as statistical tool for hospitalization duration prognosis. Another perspective could be related to the use of novel CBCT-superimposition principle, which is effective for objectification of all possible dental status changes ${ }^{29}$.

Based on the obtained results we can resume that both FISS and MFISS scores are reliable baseground parameters that could be effectively used for prediction of treatment cost and duration of hospitalization, as component parts included in non-linear insurance proceedings calculation. Greater correlation level of MFISS compare to FISS could be argumented by the orientation of such grading approach not only on the anatomical, but also on the functional evaluation of maxillofacial alterations.

Limitations of provided study related to its retrospective design, considering the use of medical patients records as primary data source, which related with risk of possible documentational errors. But such limitation was partially overcome by inclusion into study sample only cases with available X-ray diagnostic results or at least with their complete description (interpretation). Other limitation of the research is related to the relatively small study sample compare to the analogical studies provided previously. On the other hand, such situation could be argumented by the use of specific exclusion criteria, such as concomitant traumatic injury, combined trauma with related head and neck, eye-ball injury or neurotrauma and compromised anamnesis with allied somathopathologies. Neglect of such criteria potentially could help to increase the primary size of study sample, but in such situation, we would be limited in possibility to make reliable conclusion considering connection between FISS and MFISS scores with specifically maxillofacial trauma characteristics. Logically, that inclusion of patients with combined or concomitant trauma or aggravated anam- 
nesis would complicate statistical analytical approach, and deviates from originally formulated objective. Nevertheless, even considering above-mentioned limitations, obtained results demonstrated analogical pattern of relationship between FISS/ MFISS scores and economically associated rehabilitation parameters. Moreover, verified covariances could be categorized as quantitively specific for Ukrainian patients. The perspective of future study includes the statistical representation of such indices in the form of correction coefficients incorporated in the equations of the insurance indemnity amount calculation, which could be used not with ad hoc aim, but with prospective objective.

Considering limitations of provided retrospective study it could be resumed that even though FISS and MFISS scores both demonstrate reliable levels of correlation with hospitalization duration and average cost of dental rehabilitation after maxillofacial trauma injury, but MFISS approach characterized by prognostically greater level of statistical relationship with economically related treatment derivates. Moreover, differentiation capabilities of MFISS is relative greater than FISS, since independent grading of separate functional disabilities become possible. Taking this into account it could be recommended to include MFISS score as correction subcomponent or predictive factor into insurance calculation protocols during conceptual foresight of insurance coverages, or during court cases related to the assessment of dental health loss with the need of further dental rehabilitation.

\section{Financial Support}

None.

\section{Conflict of Interest}

The authors declare no conflicts of interest.

\section{REFERENCES}

1. Chen C, Zhang Y, An JG, He Y, Gong X. Comparative study of four maxillofacial trauma scoring systems and expert score. J Oral Maxillofac Surgy. 2014;72(11):2212-20. doi: 10.1016/j. joms.2014.04.035.

2. Ramalingam S. Role of maxillofacial trauma scoring systems in determining the economic burden to maxillofacial trauma patients in India. Journal of international oral health: JIOH. 2015;7(4):38

3. Teusner D, Smith V, Gnanamanickam E, Brennan D. Examining dental expenditure and dental insurance accounting for probability of incurring expenses. Community Dent Oral Epidemiol. 2017;45(2):101-11. doi: 10.1111/cdoe.12264.

4. Golkari A, Vossoughi M, Mohammadi M, Behbahanirad A, Bakhtiar M. The association between dental insurance coverage, socio-economic status and use of dental care in adolescents. J Health Sci Surveil System. 2018;6(3):149-54. doi: 10.30476/JHSSS.2019.81462.1001.

5. Chung SH. Reformation of the health insurance system: an urgent need for dental research. J Korean Acad Oral Health. 2017;41(4):229-30. Doi: 10.11149/jkaoh.2017.41.4.229.

6. Zhang J, Zhang Y, El-Maaytah M, Ma L, Liu L, Zhou LD. Maxillofacial Injury Severity Score: proposal of a new scoring system. Int J Oral Maxillofac Surg. 2006;35(2):109-14. doi: 10.1016/j.jjom.2005.06.019. 
7. Sahni V. Maxillofacial trauma scoring systems. Injury. 2016;47(7):1388-92. doi: 10.1016/j. injury.2016.02.001.

8. Sen P, Ross N, Rogers S. Recovering maxillofacial trauma patients: the hidden problems. J Wound Care. 2001;10(3):53-57. doi: https://doi.org/10.12968/jowc.2001.10.3.26062

9. Bagheri SC, Dierks EJ, Kademani D, Holmgren E, Bell RB, Hommer L, et al. Application of a facial injury severity scale in craniomaxillofacial trauma. J Oral Maxillofac Surg. 2006;64(3):408-14. doi: 10.1016/j.joms.2005.11.013.

10. Bangun $K$, Kesuma AD. Evaluation of facial trauma severity in Cipto Mangunkusumo Hospital using FISS scoring system. J Plastik Rekonstr. 2012;1(2):162-5.

11. Ramalingam $S$, Nooh N, Neelakandan R. The impact of maxillofacial trauma scoring systems in predicting maxillofacial injury severity in developing countries. International J Oral Maxillofac Surg. 2013;42(10):1232-3. doi: doi: 10.1016/j.ijom.2013.07.214.

12. Giriyan K, Kamath R, D'Souza B, Kamath S, Bhat SR. Maxillofacial injuries and its implications on economic burden in trauma victims. Medico-Legal Update. 2019;19(1):76-81. doi: 10.5958/09741283.2019.00016.1.

13. Boffano P, Roccia F, Zavattero E, Dediol E, Uglešić V, Kovačič Ž, et al. European Maxillofacial Trauma (EURMAT) project: a multicentre and prospective study. J Craniomaxillofac Surg. 2015;43(1):62-70. doi: 10.1016/j.jcms.2014.10.011.

14. Van Spall HG, Toren A, Kiss A, Fowler RA. Eligibility criteria of randomized controlled trials published in high-impact general medical journals: a systematic sampling review. JAMA. 2007;297(11):1233-40. doi: 10.1001/jama.297.11.1233.

15. General Assembly of the World Medical Association. World Medical Association Declaration of Helsinki: ethical principles for medical research involving human subjects. J Am Col Dent. 2014;81(3):14.

16. Hannigan A, Lynch CD. Statistical methodology in oral and dental research: pitfalls and recommendations. J Dent. 2013;41(5):385-92. doi: 10.1016/j.jdent.2013.02.013.

17. Shintani A. Primer of statistics in dental research: Part I. J Prosthod Res. 2014;58(1):11-6. doi: 10.1016/j.jpor.2013.12.006.

18. Giavarina D. Understanding bland altman analysis. Biochemia Med. 2015;25(2):141-51. doi: 10.11613/BM.2015.015

19. Doğan NÖ. Bland-Altman analysis: A paradigm to understand correlation and agreement. Turkish J Emerg Med. 2018;18(4):139-41. doi: 10.1016/j.tjem.2018.09.001

20. Siber S, Matijević M, Sikora M, Leović D, Mumlek I, Macan D. Assessment of oro-maxillofacial trauma according to gender, age, cause and type of the injury. Acta Stomatol Croatica. 2015;49(4):340-7. doi: $10.15644 /$ asc49/4/10

21. x Maliska MCDS, Borba M, Asprino L, De Moraes M, Moreira RWF. Oral and maxillofacial surgeryHelmet and maxillofacial trauma: a 10-year retrospective study. Braz J Oral Sci. 2012;11(2):125-9. doi: 10.20396/bjos.v11i2.8641434

22. Galvão-Moreira LV, Cantanhede ALC, de Sousa Neto AC, da Cruz MCFN. Factors affecting hospital discharge in maxillofacial trauma patients: a retrospective study. Braz J Oral Sci. 2017;16:1-10. doi: 10.20396/bjos.v16i0.8650491

23. Bocchialini G, Castellani A. Facial trauma: a retrospective study of 1262 patients. Annals Mxillofac Surg. 2019;9(1):135. doi: 10.4103/ams.ams_51_19

24. Siregar DF, Buchari FB, Tarigan UA, Lelo A. Correlation of facial injury severity scale (FISS) with length of stay, the need for surgery and the involvement of other specialists in maxillofacial trauma patients at H. Adam Malik General Hospital Medan. Global J Res Anal. 2019;8(11):8-10. doi: 10.36106/gjra 
25. Aita TG, Stabile CLP, Garbelini CCD, Stabile GAV. Can a facial injury severity scale be used to predict the need for surgical intervention and time of hospitalization? J Oral Maxillofac Surg. 2018;76(6):1280-e1. doi: 10.1016/j.joms.2018.02.002.

26. Suwal R. Analysis of Mid-face Fractures using MFISS and FISS Scoring Systems. J Nepal Dent Assoc. 2018;18(1):21-9.

27. Canzi G, De Ponti E, Novelli G, Mazzoleni F, Chiara O, Bozzetti A, et al. The CFI score: Validation of a new comprehensive severity scoring system for facial injuries. J Craniomaxillofac Surg. 2019;47(3):377-82. doi: 10.1016/j.jcms.2019.01.004.

28. Canzi G, De Ponti E, Fossati C, Novelli G, Cimbanassi S, Bozzetti A, et al. Understanding the relevance of comprehensive facial injury $(\mathrm{CFI})$ score: Statistical analysis of overall surgical time and length of stay outcomes. J Craniomaxillofac Surg. 2019;47(9):1456-63. doi: doi: 10.1016/j.jcms.2019.07.005.

29. Goncharuk-Khomyn M, Andrii K. Evaluation of peri-implant bone reduction levels from superimposition perspective: pilot study among Ukrainian implantology practice. Pesq Bras Odontoped Clin Integr. 2018;18(1):3856. doi: 10.4034/PBOCI.2018.181.10. 